\title{
O uso da inteligência de mercado como direcionador de relacionamentos chave na gestão da cadeia de suprimentos: análise de um terminal portuário
}

\author{
Murilo Vidal Branco \\ Universidad de Málaga - Departamento de Economía y Empresas \\ murilovbranco@gmail.com \\ Rafael Teixeira \\ Universidade do Vale dos Sinos - Unisinos \\ teixeira.rafa@gmail.com
}

\section{RESUMO}

Os estudos direcionados a gestão das informações vem consolidando cada vez mais o dito popular de que "o conhecimento é poder”. Afinal, quanto melhor for o suporte de informações inerentes a uma decisão, melhor ela será tomada. O presente estudo foi desenvolvido com o objetivo de gerar um método que propiciasse uma delimitação efetiva dos relacionamentos chave de uma organização através dos conceitos de inteligência de mercado. Desta forma, foram consideradas 4 etapas para a delimitação de relacionamentos chave: (i) identificação dos delimitadores estabelecidos pela própria organização (ii) proposição de pesos ponderados a cada delimitador de relacionamento estratégico (iii) validação do método proposto e do sistema de pesos (iv) aplicação dos parâmetros desenvolvidos aos clientes da organização. O método proposto foi aplicado empiricamente em um terminal de containers e a partir dos resultados apresentados percebe-se a contribuição do artigo no desenvolvimento de delimitadores padronizados de relacionamentos chave voltado a organização.

Palavras-chave: Gestão de Relacionamento Chave. Inteligência de Mercado. Gestão Portuária.

\begin{abstract}
The information management studies has increasingly consolidate the popular saying that "knowledge is power". After all, the better the support of information inherent in a decision, the better it will be taken. The present study was developed with the aim of generating a method that propitiates an effective delineation of the key relationships in an organization based on the concepts of Market Intelligence. Thus, we considered 4 steps for defining key relationships: (i) identification of the boundaries established by the organization itself (ii) proposing weighted weights for every strategic relationship delimiter (iii) validation of the method and system of weights (iv) application of parameters developed the organization's clients. The proposed method was applied empirically in a container terminal and from the presented results we saw the creation of standardized key relationships delimiters facing this organization.
\end{abstract}

Keywords: Key Relationship Management. Market Intelligence. Ports Management. 


\section{Introdução}

Estudos sobre gestão da cadeia de suprimentos vêm trazendo importância a gestão orientada à cadeia de suprimentos (TONGZON et al., 2009; ESPER et al., 2010; STANK et al., 2011; OMAR et al., 2012). Esta abordagem apresenta que as organizações devem dimensionar suas estratégias de gestão além do seu cliente final e coordenar suas ações de integração entre os membros de sua cadeia para uma maior eficiência (MENTZER et al., 2001; HULT et al., 2008; LIN et al., 2010). É praticamente inviável que grandes organizações consigam manter as mesmas ações estratégicas, com alta intensidade, a todos os seus clientes ou membros de sua cadeia. Além de normalmente não haver recursos financeiros e humanos para tal, é provável que a excelência na integração a todos os membros da cadeia possa ocasionar em desperdícios de esforços que poderiam ser direcionados com maior intensidade a outros membros de maior relevância. Isto é, podem existir trade-offs (SKINNER, 1969; HAYES; WHEELWRIGHT, 1984) na gestão da cadeia de suprimentos no que tange aos relacionamentos de uma empresa focal.

Essa discussão mostra como o tema ligado aos relacionamentos na cadeia de suprimentos tem sido recorrente na literatura da área (MALONI; BENTON, 1997; CAO; ZHANG, 2011; CORSTEN et al., 2011; TEIXEIRA; LACERDA, 2010). Porém pouco tem sido feito para ajudar as empresas a determinar quais parceiros devem ser o foco de atenção. A correta definição dos relacionamentos chave das organizações torna-se, portanto, relevante para a gestão, pois a partir deste refinamento poderão ser direcionados os esforços de gestão de cada organização e, por sua vez, a melhor utilização dos recursos financeiros, humanos e do alcance de competitividade. Ou seja, existe uma combinação ótima entre os parceiros que uma empresa considera importantes e o tipo de relacionamento que a mesma deve ter com esses parceiros. Essa abordagem, sobre uma combinação ótima de atividades e recursos, têm sido recorrente na literatura de gestão de operações e serviços (HAYES; WHEELWRIGHT, 1984; HUETE; ROTH, 1988; STOCK; TATIKONDA, 2008). Em meio a esse debate sobre a gestão da cadeia de suprimentos e dos relacionamentos chave que a envolvem surge a questão: como determinar quais os relacionamentos chave mais importantes para a gestão da cadeia de suprimentos de uma determinada empresa?

Neste trabalho, entende-se que a inteligência de mercado é uma ferramenta que pode auxiliar na delimitação do foco estratégico das organizações. Como parte deste processo, o direcionamento dos relacionamentos chave inclui a definição dos principais membros da cadeia de suprimentos e a respectiva atuação estratégica de acordo com o apanhado de informações internas e externas que circundam a organização. Estudos sobre inteligência de mercado vêm consolidando a idéia de que cada vez mais as organizações precisam aprofundar as técnicas de gestão das informações que a cercam em seu dia-a-dia (KOHLIL; JAWORSKI, 1990; LACKMAN et al., 2000; WEE, 2001, HANNULA; PIRTTIMÄKI, 2003; SABHERWAL; BECERA-FERNANDEZ, 2011; CUSTIS, 2012).Da mesma forma que outros setores da empresa devem-se voltar ao mercado, a gestão da cadeia de suprimentos também o deve (MIN et al., 2007).

O próprio gerenciamento das informações de forma estruturada vem sendo tema de pesquisas ao longo dos anos por meio de diferentes abordagens (LACKMAN et al., 2000; WEE, 2001; DISHMAN; CALOF, 2008). Todavia, o objetivo da administração destes dados, ainda que com diferentes pontos de vista, é de aprimorar as técnicas de governança e facilitar a tomada de decisões. Desde o cruzamento de dados retirados dos ambientes externo e interno, destacado por Porter (1984), as informações do sistema que envolve as organizações são tidas como fundamentais para a estruturação de estratégias que direcionem sua vantagem competitiva.

O objetivo, portanto, do presente estudo é apresentar um método estruturado para o direcionamento de relacionamentos chave da cadeia de suprimentos em uma organização. Para isso, serão utilizados os procedimentos e diretrizes da inteligência de mercado. O método apresentado foi testado no setor portuário, mais precisamente em um terminal de containers, já que existe um consenso entre especialistas da área que existe necessidade de aprimorar a eficiência da logística no Brasil. O setor portuário vem recebendo atenção do atual governo com medidas de incentivo ao investimento privado e tentativas de amenizar os gargalos de infra estrutura. Este momento atual, em que o crescimento econômico do país enfrenta problemas de escoamento de sua produção, justificaria por si só a escolha de um terminal de containers para teste do modelo proposto neste trabalho.

Uma contribuição desse trabalho é apresentar um método para determinação dos relacionamentos chave na gestão da cadeia de suprimentos. Esse método pode ser útil para a discussão sobre orientação à cadeia de suprimentos e para toda literatura sobre relacionamentos na cadeia de suprimentos, apresentando uma forma de lidar com essas questões na prática. Outra contribuição desse artigo é o fato de que o mesmo aborda quase todos os eles de cadeia de suprimentos, já que a maioria dos trabalhos em gestão de cadeia de suprimentos proporciona dados e análises de uma empresa focal na cadeia ou, em alguns casos, de uma díade ou tríade. 
As seções à seguir apresentam uma revisão da literatura, apresentando os conceitos de inteligência de mercado e o desenho da cadeia em que a empresa enfoque do estudo está inserida. Posteriormente é explicado o método, proposta central do estudo, e os resultados.

\section{Inteligência de mercado}

A inteligência de mercado (IM) tem um papel mais amplo do que simplesmente atender as necessidades e preferências dos usuários. Conforme Sabherwal e Becera-Fernandez (2011), IM é considerada como a arte de ganhar vantagem competitiva através dos dados, e abrange áreas como 'Análise do Negócio', 'Relatórios Empresariais’ e ‘Gestão de Desempenho’. É vista como ferramenta essencial para possibilitar às empresas a oportunidade de estar à frente da concorrência, segundo Wee (2001). O autor ainda argumenta que o desenvolvimento de inteligência de mercado é um processo contínuo e sistemático de busca e análise de informações, sendo guiado por resultados e de acordo com a estratégia da empresa. Inteligência de mercado é primeiramente um processo qualitativo, explorando fontes secundárias e primárias abrangendo uma variedade ampla de redes de organizações para a coleta de informações, como: consumidores, concorrentes, fornecedores, colaboradores da organização e ainda base de dados eletrônicos e mídia em massa. Um sistema efetivo de inteligência de mercado reduz o tempo usado para a tomada de decisão através do aprimoramento da informação (HANNULA; PIRTTIMÄKI, 2003).

As fontes de dados mais utilizadas para alimentação da inteligência de mercado são classificadas por Lackman et al. (2000) como internas e externas à organização. Os autores desenvolvem uma pesquisa empírica entre empresas que utilizam a ferramenta e ranqueiam as 18 fontes sugeridas. O resultado é apresentado na tabela 1.

Tabela 1 - Ranking das fontes de dados da inteligência de mercado.

\begin{tabular}{|l|l|}
\hline \multicolumn{1}{|c|}{ Fonte de dados } & \multicolumn{1}{c|}{$\begin{array}{c}\text { Percentual } \\
\text { de } \\
\text { utilização }\end{array}$} \\
\hline \multicolumn{1}{|c|}{ Internos } & \\
\hline 1. Consumidores & 100 \\
\hline 2. Fábricas & 100 \\
\hline 3. Pesquisa e desenvolvimento & 100 \\
\hline 4. Força de vendas & 78 \\
\hline 5. Evidências físicas (produto ou protótipo) & 64 \\
\hline 6. Cotas de vendas & 58 \\
\hline 7. Histórico de vendas & 50 \\
\hline 8. Feiras e eventos & 48 \\
\hline 9. Novas contratações & 43 \\
\hline
\end{tabular}

\begin{tabular}{|l|l|}
\hline \multicolumn{1}{|c|}{ Fonte de dados } & \multicolumn{1}{|c|}{$\begin{array}{c}\text { Percentual } \\
\text { de } \\
\text { utilização }\end{array}$} \\
\hline \multicolumn{1}{|c|}{ Externos } & \\
\hline 1. Reunião com clientes & 100 \\
\hline 2. Distribuidores & 68 \\
\hline 3. Consumidores & 67 \\
\hline 4. Negócios associados & 58 \\
\hline 5. Projetos de pesquisa de marketing & 51 \\
\hline 6. Fornecedores & 45 \\
\hline 7. Serviços on line & 44 \\
\hline 8. Periódicos (revistas, jornais) & 40 \\
\hline 9. Publicações governamentais & 33 \\
\hline
\end{tabular}

Fonte: LACKMAN, C.; SABAN, K.; LANASA, J. The contribution of market intelligence to tactical and strategic business decision. Marketing intelligence Planning, 2000 v. 18, n. 1, p. 6-8.

As informações administradas pela inteligência de mercado geram suporte não somente para a determinação da estratégia da empresa (DISHMAN; CALOF 2008), mas também para a definição dos relacionamentos chave da cadeia. Os relacionamentos chave (ou estratégicos) são aqueles delimitados pela estratégia de cada empresa como relevantes para sua organização. Estas podem ser membros da cadeia que geram um bom resultado para o negócio ou ainda membros críticos para o fluxo das atividades da rede em que estão inseridas. Pardo (2001) sugere que a gestão de relacionamentos chave passa pela criação de novas práticas e a sua integração com a estrutura existente. As novas práticas envolvem coordenar as informações e ações da cadeia em tempo e espaço em relação ao cliente em sua integridade. Gerenciamento de relacionamento chave na cadeia de suprimentos é definido por Ivens et al. (2009) como a administração de relacionamento com membros da cadeia que a organização tenha identificado como importantes com relação a sua estratégia. A eficiência da orientação à cadeia de suprimentos é fortemente vinculada à gestão dos membros chave da cadeia de suprimentos, segundo Miocevic e Crnjak-Karanovic (2012)

Portanto, sugere-se que quanto melhor for a qualidade da inteligência de mercado de uma organização, melhor delimitado estarão os relacionamentos chave da sua cadeia de suprimentos. O constante cruzamento das informações externas e internas delimitará a relevância de cada membro da cadeia, dimensionando o nível estratégico de relacionamento a ser direcionado a cada um. Estas delimitações sofrerão variações ao longo do tempo de acordo com o dinamismo da própria cadeia e do ambiente externo a ela. 


\section{Desenho da cadeia de suprimentos nos portos}

Um dos elementos essenciais para a gestão da cadeia de suprimentos e aplicação eficaz da Inteligência de Mercado é o entendimento e conhecimento explícito de como a estrutura da cadeia de suprimentos é configurada. Conforme Lambert (1998), três aspectos estruturais primários precisam ser levados em consideração: (i) os membros da cadeia de suprimentos, (ii) as dimensões estruturais da rede de relacionamento e (iii) os diferentes tipos de processos que conectam cada elo a cadeia.
Portanto, é preciso entender as engrenagens da cadeia para posteriormente identificar os seus relacionamentos chave. Em seus estudos sobre definição da cadeia de suprimentos, Mentzer et al. (2001) apresentam três níveis de cadeia de suprimentos: (a) cadeia de suprimentos direta, consistindo em organização, fornecedor e cliente; (b) cadeia de suprimentos estendida, incluindo intermediários podendo aparecer em ambas as pontas; e (c) cadeia de suprimentos completa, inclui todas as organizações envolvidas nas trocas e fluxos de produtos, serviços, finanças e informações (Figura 1).

Figura 1 - Tipos de canal de relacionamento.

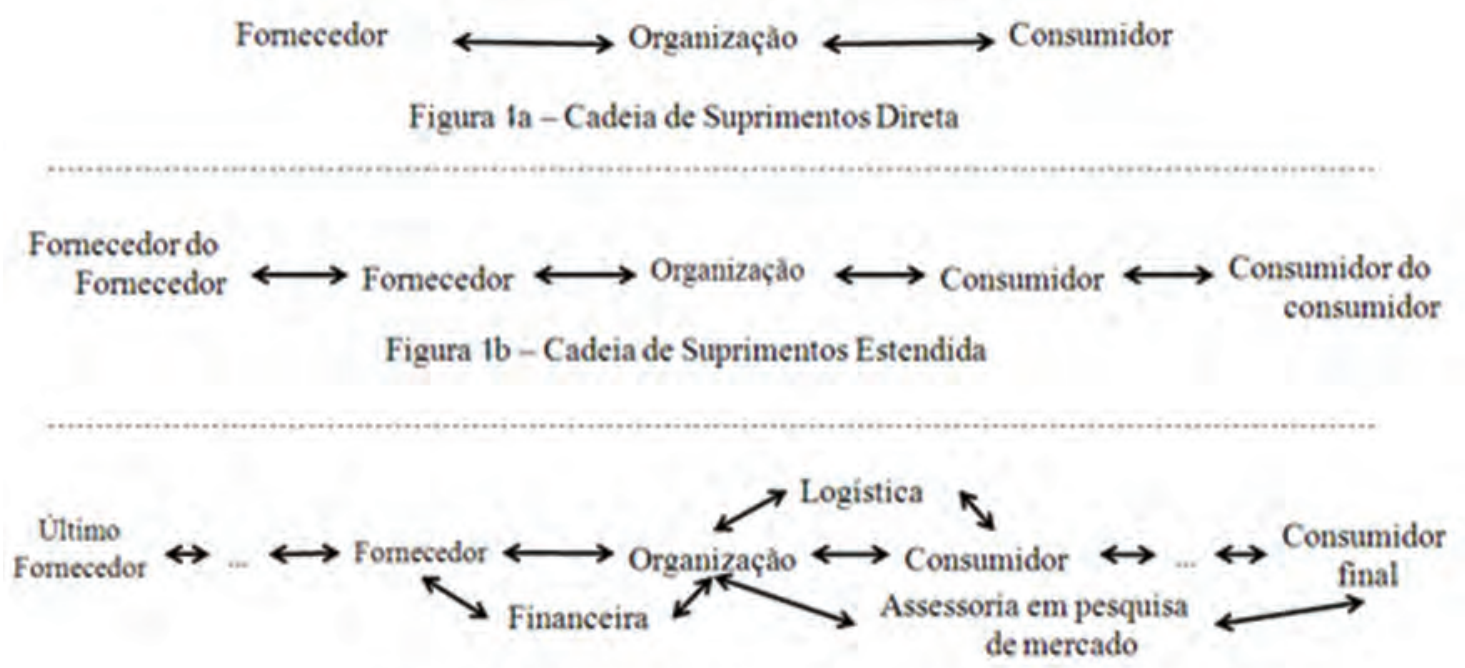

Figura IC - Cadeia de Suprimentos Completa

Fonte: MENTZER, J. T.; KEEBLER, J. S.; NIX, N. W.; SMITH, C. D.; ZACHARIA, Z. G.

Defining supply chain management. Journal of Business Logistics, 2001 v. 22, n. 2, p. 1-25.

O reconhecimento do gerenciamento da cadeia de suprimentos como medidor de competitividade nas abordagens tradicionais sobre o tema, não são suficientes para descrever e interpretar a integração dinâmica do mercado portuário atual (DE MARTINO; MORVILLO, 2008). Como resultado desta constatação, uma nova visão do sistema vem se afirmando. Apesar de a competitividade de um porto se manter fortemente vinculada às variáveis de tipo e estrutura (economia local, modelo institucional e infra-estrutura de conexão), De Martino e Morvillo (2008) sugerem que cada vez mais cresce a dependência de sistemas informatizados utilizados para as trocas de informações entre os atores da mesma e de outras comunidades portuárias.
Para melhor identificar o comportamento dos elementos da rede da cadeia portuária, De Martino e Morvillo (2008) utilizam o modelo de Dubois et al (2003). Segundo os autores, o modelo apresentado na figura 2 foi desenvolvido para uma cadeia específica de negócios, podendo ser readaptado a qualquer cadeia, conforme sua estrutura.

Devido a interdependência dos membros da cadeia, Dubois et al (2003) argumentam que é necessário entender o contexto global em que as organizações se encontram. Os autores levam em consideração que contratempos entre organizações interdependentes dentro de uma mesma cadeia impactam no todo, como em um efeito dominó. Por este motivo, Dubois et al (2003) afirmam que através de uma maior integração as organizações podem buscar aumento da eficiência, criando valor à cadeia. 


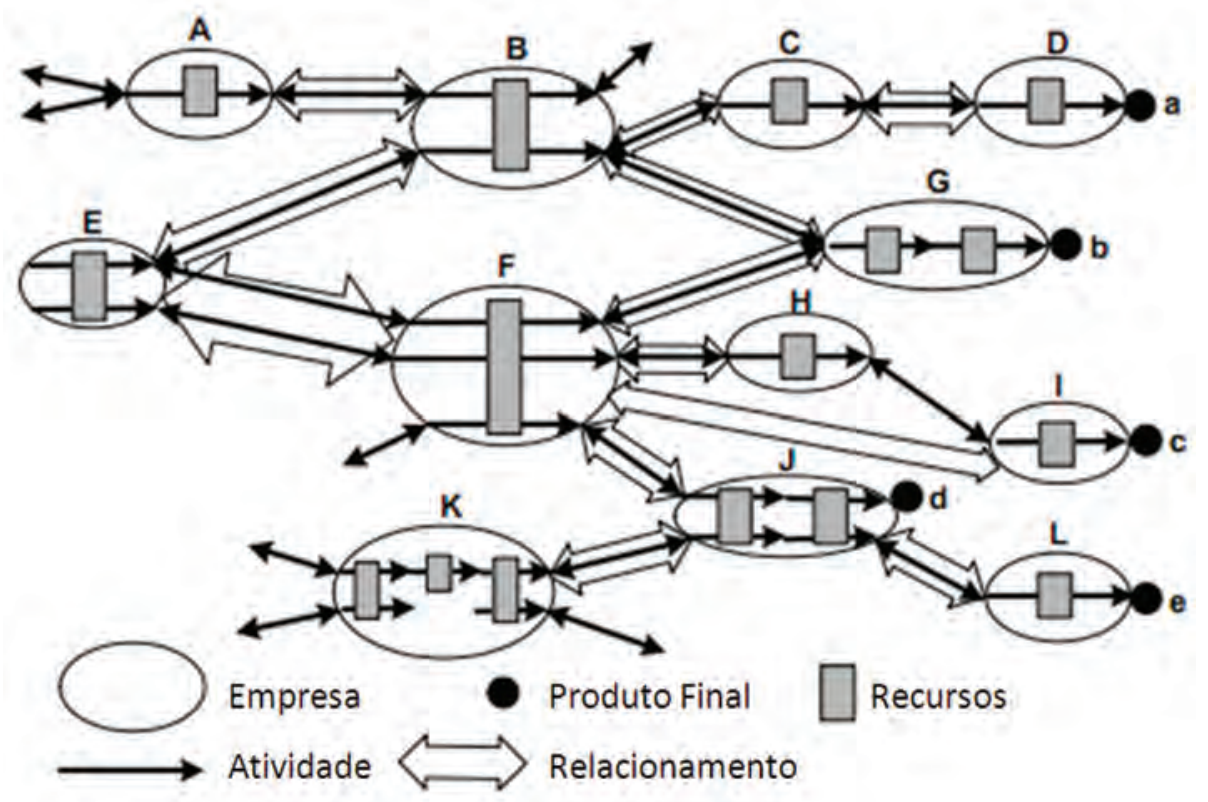

Fonte: DUBOIS, A.; HULTHÉN, K.; PEDERSEN, A. Interdependence Within and Among 'Supply Chains'. Artigo apresentado no 12nd Internacional IPSERA Conference, Budapest, 2003.

Levando em consideração os pontos levantados no modelo de Dubois et al (2003), De Martino e Morvillo (2008) aplicaram o modelo no contexto da operação portuária. A figura 3 apresenta o modelo adaptado pelos autores, desenhando a rede de acordo com os elos específicos da cadeia portuária.

No modelo proposto por De Martino e Morvillo (2008, pg. 583), o "porto é representado pela rede de atores que carregam um número de atividades na cadeia de em uma colaboração estreita, dividindo diferentes recursos. Quanto maior o nível de colaboração (integração) entre os membros, maior serão os benefícios percebidos”. Neste caso, as empresas A e B exportam para as empresas D e E através do Porto X, sendo FF - Freight Forwarder, S - a companhia marítima (Shipping Company); LO-operador logístico, TO - Terminal de Container, $\mathrm{H}$ - agentes de carga e traders.

Figura 3 - Criação de valor do porto ' $X$ ’ através da rede de suprimentos simplificada.

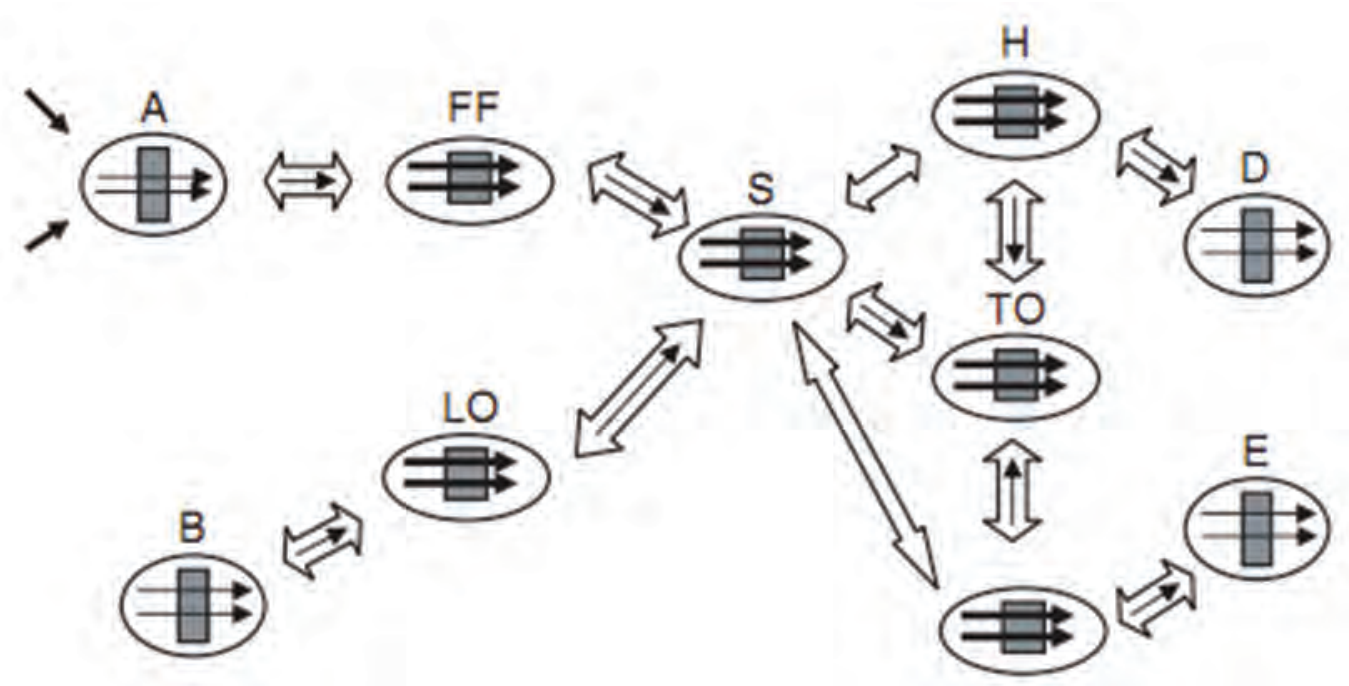

Fonte: DE MARTINO, M.; MORVILLO, A. Activities, resources and inter-organizational relationships: key factors in port competitiveness. Maritime Policy \& Management, 2008 v. 35, n. 6, p. 571-589. 
As atividades desenvolvidas por estes atores foram divididas em 3 macrocategorias, de acordo com De Martino e Morvillo (2008):

1. Atividades relacionadas a internacionalização (transporte marítimo e acesso marítimo).

2. Atividades relacionadas ao porto (transbordo, armazenagem, valor agregado a logística, manuseio e distribuição).

3. Atividades relacionadas ao transporte interno (Transporte rodoviário, ferroviário e navegação interior).

O porto pode ser considerado como um membro da cadeia de suprimentos, porém, no presente estudo, o porto é considerado como um cluster de organizações, seguindo a definição de Carbone e De Martino (2003), onde estão inseridos os terminais de container e outros operadores logísticos e de transporte, formando a cadeia de suprimentos específica. A estrutura da cadeia portuária final desenvolvida neste capítulo é utilizada como base no presente estudo, com o objetivo de delimitar a cadeia de suprimentos portuária, assim como as suas macrocategorias de atividades.

\section{Método}

Lackman (2000), Wee (2001), Hannula e Pirttimäki (2003), Sabherwal e Becera-Fernandez (2011), Custis (2012) concordam que a inteligência de mercado é um processo qualitativo, através da exploração de fontes secundárias e primárias abrangendo uma variedade ampla de redes de organizações para a coleta de informações internas e externas à organização. O método proposto neste trabalho visa identificar os relacionamentos chave da organização de acordo com fatores delimitadores que são definidos através de Inteligência de Mercado. Para desenvolvimento do método foram analisados fatores externos e internos a organização relevantes aos clientes da organização. Para organização do passo a passo do método proposto, foram determinadas 3 etapas de evolução. (i) identificação dos delimitadores estabelecidos pela própria organização, através de entrevista realizada com colaboradores do Departamento Comercial do Tecon Rio Grande (2012); (ii) proposição de pesos ponderados a cada delimitador de relacionamento estratégico para alocação dos membros da cadeia em sua respectiva categoria de relacionamento estratégico; e (iii) validação do método proposto e do sistema de pesos desenvolvido junto ao Departamento Comercial do Tecon Rio Grande (2012) para validação dos parâmetros. (iv) aplicação dos parâmetros desenvolvidos aos clientes da organização. Na figura 4 são apresentados os passos do método proposto.
Figura 4 - Método de Delimitação de Relacionamentos Chave.

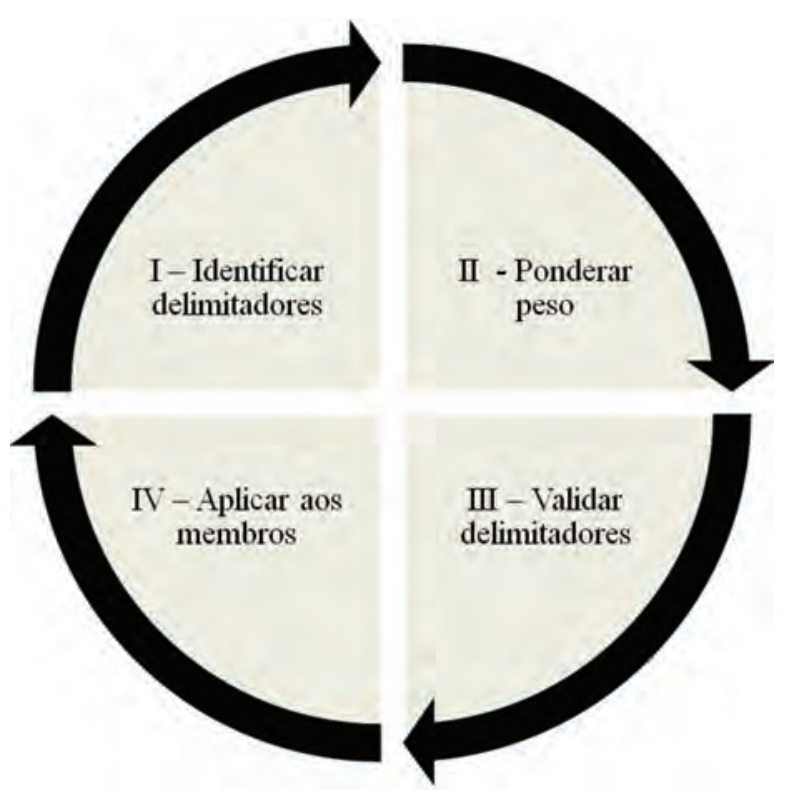

Fonte: Elaborado pelo autor (2013).

O método apresentado na figura 4 apresenta um fluxo contínuo de análise das informações por meio da inteligência de mercado. Os delimitadores podem variar ao longo do tempo de acordo com o dinamismo do mercado. Após identificação e ponderação de pesos aos delimitadores é preciso validar internamente em forma de discussão as informações finais antes da aplicação aos clientes da organização.

\subsection{Identificação dos Delimitadores Estratégicos}

Nesta primeira etapa é realizada uma análise sobre os ambientes externos e internos da organização pela óptica da estratégia da empresa. São analisadas as oportunidades e ameaças inerentes a organização e a partir desta análise são definidos aspectos relacionados aos clientes, ou aos membros da cadeia, que sejam cruciais para o bom desempenho da organização. Nesta primeira etapa é primordial que a organização tenha conhecimento dos seus objetivos e as estratégias para alcançá-los. Sabherwal e Becera-Fernandez (2011) ressaltam a importância da IM para o desenvolvimento da estratégia da organização.

As informações internas e externas (LACKMAN et al., 2000) devem ser cruzadas com a estratégia da organização e a relevância de cada item são avaliadas conforme os objetivos da organização e a partir desta etapa são definidos os delimitadores estratégicos a serem analisados na segunda etapa. Portanto, neste primeiro momento deve-se analisar as informações dos ambientes internos e externos e definir os delimitadores importantes para a estratégia da organização. 


\subsection{Criação de pesos ponderados}

De acordo com a enumeração de delimitadores estratégicos definidos pela organização na primeira etapa do processo, são identificados aqueles que possuam maior relevância em relação aos outros. Assim como na primeira etapa, este ranking de relevâncias deverá ser suportado pelos objetivos e estratégias da organização.

Os delimitadores levantados na primeira etapa devem ser colocados frente a frente com a estratégia da organização. De acordo com o grau de ameaça ou oportunidade do delimitador estratégico, este deverá ter um peso de igual relevância. Por exemplo, se uma organização tem como objetivo alcançar 100\% do market share de determinada região, a utilização dos serviços ou produtos de um concorrente por um cliente ou membro da cadeia pressupõe a definição de um peso de maior relevância, pois ações estratégicas de maior força deverão ser aplicadas a este cliente. Assim por diante, os pesos auferidos irão nortear a importância relativa de cada delimitador para o desenvolvimento da estratégia.

\subsection{Validação dos delimitadores estratégicos}

A partir da classificação dos delimitadores estratégicos definidos no processo anterior, é preciso validar as informações levantadas nas etapas anteriores e, por consequência validar o método proposto. Nesta etapa, para identificação da propriedade correta dos pesos aplicados a cada delimitador é a elaboração de um brainstorming entre os gestores da organização. Com base no comum acordo entre os gestores é possível potencializar a efetividade dos delimitadores apresentados na etapa anterior e assegurar que os pesos e delimitadores sugeridos estão de acordo com a estratégia da organização.

Como o processo é estratégico para a organização, pois definirá o rumo dos recursos e esforços de toda a organização, é importante que pessoas influentes e tomadoras de decisões estejam envolvidas nesta fase do processo. $\mathrm{O}$ maior refinamento dos pesos ponderados e da relevância das informações é desenvolvido nesta etapa.

\subsection{Aplicação dos delimitadores aos membros da cadeia (ou clientes)}

Em posse dos pesos ponderados por cada delimitador de relacionamento chave, é preciso definir uma base de cálculo para a aplicação. Igualmente, esta base de calculo será definida conforme objetivos da organização e suas estratégias para alcançá-los. Exemplos de aplicação podem ser a quantidade de produtos adquiridos ou o faturamento de cada cliente ou membro da cadeia. Os índices de relacionamento chave serão definidos a partir do resultado entre o peso ponderado multiplicado pela base de cálculo determinada. Desta forma, estarão ranqueados os clientes chave da organização e a partir desta avaliação a aplicação de recursos poderá ser direcionada de acordo com a relevância apontada pela sua delimitação.

A aplicação empírica do método proposto é apresentado a seguir, desenvolvido em um terminal portuário.

\section{Aplicação do método}

Nesta etapa foi desenvolvida a aplicação das 4 fases do método proposto na cadeia portuária do terminal portuário escolhido para o estudo. Conforme apresentado na revisão da literatura, é imprescindível entender o desenho da cadeia em que a organização está inserida. Assim sendo, o terminal portuário possui como agentes principais os Clientes de Carga, os Agentes de Carga, os Operadores Logísticos e os Armadores.

Os níveis estratégicos estabelecidos para cada membro foram desenvolvidos pelos delimitadores estratégicos após análise das entrevistas realizadas com membros do Departamento Comercial do Tecon Rio Grande (2012). A partir desta análise desenvolveu-se um sistema de padronização dos níveis estratégicos de relacionamento. A seguir são apresentados os resultados segregados por cada elo da cadeia e apresentando a evolução da primeira fase do método proposto: a identificação dos delimitadores estratégicos.

\subsection{Fase I - clientes de carga}

Os clientes de carga (CC) da cadeia portuária são considerados os embarcadores e desembarcadores que movimentam suas cargas através do porto. Esses atores podem ser tanto exportadores, importadores como também usuários da cabotagem (operação de transporte marítimo realizado entre portos do próprio país). Os CCs são considerados os proprietários da carga, tanto na venda como na compra, e as cargas estão vinculadas em seus nomes.

Com mais de 2500 clientes de carga cadastrados, em entrevista não estruturada com funcionários do departamento comercial do Tecon Rio Grande definiram-se os delimitadores estratégicos deste elo: (i) faturamento, (ii) quantidade de containers movimentados, (iii) se os containers movimentados são refrigerados, (iv) distância rodoviária entre a planta produtora e os portos concorrentes, (v) se mantém operações com terminais concorrentes e (vi) se utilizam acesso intermodal ou se é viável sua utilização. A delimitação por faturamento determina que a categoria A são as empresas que compõem 40\% do faturamento, enquanto que a categoria B são as organizações que concentram o faturamento entre $40 \%$ e $60 \%$ e a categoria C entre $60 \%$ e $100 \%$. Com relação a 
quantidade de containers movimentados, consideram-se mais estratégicos aqueles clientes que movimentam acima de 100 containers mensais, ou mais de 50 containers refrigerados ao mês. De nível intermediário, são aqueles que movimentam entre 10 e 99 containers/mês e, no nível de monitoramento, aqueles que movimentam menos que 10 containers mensais. Esta relação pode ser reajustada caso o segundo item delimitador seja constatado: que trata sobre carga refrigerada. Devido a maior geração de receita deste tipo de carga, em função do abastecimento energético durante a sua armazenagem, estes clientes tem relevância estratégica maior.

Outro fator determinante da relevância estratégica do CC para o Tecon RG é a sensibilidade da empresa com relação à distância rodoviária comparada entre sua planta, os portos concorrentes e o Porto de Rio Grande. A logística de acesso ao porto pode ser determinante para a utilização de um terminal ou outro, portanto clientes com proximidade maior para os portos concorrentes são considerados com nível estratégico mais relevante para o Tecon RG, e da mesma forma aqueles que também realizam operações pela concorrência. A utilização da intermodalidade (acesso ferroviário ou navegação interior pela Lagoa dos Patos) também é relevante para a análise da categoria estratégica. As empresas que utilizam estes modais trabalham exclusivamente com o Tecon Rio Grande, visto que em relação a concorrência local, o terminal foco do estudo é o único que possui estrutura de interconexão entre os modais rodoviário, ferroviário e hidroviário.

\subsection{Fase I- agentes de carga}

Conforme o Departamento Comercial do Tecon Rio Grande (2012), os agentes de carga (AC) são definidos como prestadores de serviço que normalmente se encarregam de parte ou totalidade da operação logística do cliente de carga, intermediando negociações com armadores, operadores logísticos e terminais de container, de acordo com a necessidade do cliente. Os agentes de carga podem se responsabilizar por todos os serviços necessários para levar a carga de um cliente de carga ao outro, como também podem ser contratados para realizar somente algumas partes do processo, dependendo da estrutura do agente e de seu cliente de carga.

O Tecon Rio Grande trabalha com mais de 140 agentes de carga, sendo que os delimitadores estratégicos definidos pela empresa focal para este tipo de elo são: (i) a quantidade de containers movimentados por cada agente, e (ii) se mantém operações com terminais concorrentes. Com relação a quantidade de containers movimentados, aqueles responsáveis por 100 ou mais containers ao mês são considerados mais relevantes para a estratégia da organização, ou seja, no nível de criação de soluções. Os responsáveis pelo embarque e desembarque de
10 a 99 containers mensais são considerados de nível intermediário e aqueles que movimentam menos de 10 containers/mês, são considerados clientes do nível de monitoramento. Com relação a movimentação com terminais concorrentes, avalia-se o nível estratégico pela existência da movimentação de containers também por outros portos e a relevância deste volume.

\subsection{Fase I - armadores}

As companhias marítimas, responsáveis pelas embarcações e por disponibilizar os containers aos clientes de carga, são também conhecidas como armadores (Ar). Sendo um dos principais componentes da cadeia portuária, os armadores podem definir em qual porto atracar e influenciar diretamente na logística de todo o resto da cadeia.

No Tecon Rio Grande, conforme levantamento realizado junto ao seu Departamento Comercial (2012), 13 companhias marítimas operam neste terminal. Para dimensionar sua relevância estratégica são avaliados: (i) quantidade de containers movimentados ao mês, (ii) quantidade de serviços (linhas) oferecidas por semana no Tecon, e (iii) se oferecem serviços similares nos portos concorrentes. Foram considerados membros de nível estratégico mais elevado, os armadores que embarcam e desembarcam, somados, mais de 1000 containers cheios ao mês, como nível intermediário, os que movimentam entre 100 e 999 containers cheios ao mês e como nível estratégico inferior, aqueles que movimentam abaixo de 100 containers mês.

Sobre a análise de serviços oferecidos, são considerados armadores estratégicos da cadeia do terminal, aqueles que possuem 2 ou mais serviços semanais, devido a maior frequência de atracação. Também foram considerados estratégicos aqueles armadores que oferecem serviços similares em outros portos, pois este fato permite que a venda do serviço influencie a escolha do terminal de containers a ser utilizado.

\subsection{Fase I - operadores logísticos}

São considerados operadores logísticos (OL) no presente estudo, os prestadores de serviço rodoviário e ferroviário que interligam a carga entre o Tecon Rio Grande e as plantas dos clientes de carga. Os custos gerados pelos operadores logísticos são determinantes para o direcionamento de uma carga a um porto ou outro e por isso são elos fundamentais na composição da cadeia portuária.

O Tecon Rio Grande trabalha com mais de 318 operadores logísticos sendo utilizado como delimitadores estratégicos, os seguintes aspectos: (i) quantidade de containers movimentados e (ii) se mantém operações 
com portos concorrentes. Com relação a quantidade de containers movimentados, são considerados estrategicamente relevantes, aqueles operadores logísticos que movimentam acima de 300 containers/mês. Em um nível intermediário de relevância estratégica, são considerados aqueles OLs responsáveis pela movimentação entre 10 e 299 containers mensais, enquanto os de menor relevância são aqueles que movimentam menos que 10 containers mês. Aqueles operadores logísticos que possuem operações com portos concorrentes possuem maior relevância estratégica, pois podem influenciar na opinião dos clientes de carga na escolha do terminal de containers.

\subsection{Fase II e III - ponderação dos pesos e validação dos dados}

A partir da identificação dos delimitadores apresentados nos sub capítulos anteriores, desenvolveu-se um esquema de peso relativo para cada. As medidas sugeridas foram apresentadas ao Departamento Comercial do Tecon Rio Grande e após brainstorming com gestores deste setor (gerência e diretoria), foram realizados alguns ajustes na força dos pesos aferidos além de novas definições adicionadas. Desta forma, obteve-se um padrão final de delimitadores estratégicos, conforme é possível identificar na tabela 2.

Tabela 2 - Delimitadores estratégicos.

\begin{tabular}{|c|c|c|c|c|c|}
\hline Definição & Peso Auferido & CC & AC & OL & Ar \\
\hline \multicolumn{6}{|l|}{ Faturamento } \\
\hline Até $40 \%$ do faturamento & Cliente A & $\mathrm{x}$ & & & \\
\hline De $40 \%$ a $60 \%$ do faturamento & Cliente B & $\mathrm{x}$ & & & \\
\hline De $60 \%$ a $100 \%$ do faturamento & Cliente C & $\mathrm{X}$ & & & \\
\hline \multicolumn{6}{|l|}{ Movimentação de containers } \\
\hline Número de containers movimentados & 1 & $\mathrm{x}$ & $\mathrm{x}$ & $\mathrm{x}$ & $\mathrm{x}$ \\
\hline Containers refrigerados movimentados & 1,5 & $\mathrm{X}$ & & & \\
\hline \multicolumn{6}{|l|}{ Intermodalidade } \\
\hline Usuário atual de intermodalidade & 1,5 & $\mathrm{x}$ & & & \\
\hline É viável utilizar a intermodalidade & 1,3 & $\mathrm{X}$ & & & \\
\hline \multicolumn{6}{|l|}{ Distância em relação ao concorrente } \\
\hline a favor do Terminal & 1 & $\mathrm{x}$ & & & \\
\hline até 100 km pró concorrência & 1,3 & $\mathrm{X}$ & & & \\
\hline mais de 100 km pró concorrência & 1,5 & $\mathrm{X}$ & & & \\
\hline \multicolumn{6}{|l|}{ Serviços na concorrência } \\
\hline Não utiliza serviço do concorrente & 1 & $\mathrm{x}$ & $\mathrm{x}$ & $\mathrm{x}$ & $\mathrm{x}$ \\
\hline Utiliza serviço do concorrente & 1,5 & $\mathrm{X}$ & $\mathrm{x}$ & $\mathrm{x}$ & $\mathrm{x}$ \\
\hline \multicolumn{6}{|l|}{ Serviços (linhas) oferecidos no terminal } \\
\hline Serviço não regular & 1 & & & & $\mathrm{x}$ \\
\hline 1 Serviço regular/semana & 1,3 & & & & $X$ \\
\hline 2 ou mais Serviços regulares/semana & 1,5 & & & & $x$ \\
\hline
\end{tabular}

Fonte: Elaborado pelo autor (2012).

Legenda: CC - Clientes de Carga; AC - Agentes de Carga; OL - Operador Logístico; AR - Armadores.

\subsection{Fase IV - aplicação aos membros da cadeia}

Os pesos atribuídos a cada item delimitador foram multiplicados ao número de containers movimentados por cada cliente, pois esta foi a base de cálculo sugerida para aplicação durante brainstorm na fase anterior desenvolvida para a validação dos pesos sugeridos aos gestores. Com este índice foi possível dimensionar uma alocação de importância estratégica para cada membro da cadeia, subdividindo-a em clientes de categoria A,
B e C, conforme seu respectivo nível de importância estratégica para o terminal.

O terminal foco do estudo possui mais de 3000 parceiros cadastrados em seu banco de dados (DEPARTAMENTO COMERCIAL TECON RIO GRANDE, 2012). Porém, diversos membros continuam cadastrados mesmo tendo movimentado apenas 1 container ao ano, não sendo este usuário esporádico relevante para este estudo. Portanto, o modelo do sistema de pesos apresentado foi aplicado 
aos membros da cadeia do terminal de containers escolhido, com a exceção dos membros com movimentação menor que 10 containers no ano, ou seja, menos que 1 container ao mês, que foram excluídos na análise desta etapa. Assim sendo, a avaliação foi realizada a 1441 membros da cadeia portuária do terminal, alocados em suas respectivas categorias, conforme tabela 3.

A tabela 3 apresenta o resultado final deste estudo, delimitando um parâmetro para relacionamentos chave de um terminal de containers. Na categoria A, das 1441 organizações ativas na cadeia portuária do terminal, um total de 112 membros deverão receber ações prioritárias por parte do terminal. Estes membros são responsáveis, em uma média ponderada, por 76\% das cargas movimentadas no terminal portuário. Da mesma forma, a categoria B é representada por 298 membros que representam 19,25\% do total de movimentação. Ações direcionadas a esses membros também são relevantes para a gestão do negócio, porém com menor intensidade em comparação com clientes da categoria A. Por fim, os membros da categoria C são 1031 organizações, que, apesar do elevado número de empresas, representam apenas $4,75 \%$ do volume total de containers movimentados pelo terminal.

Tabela 3 - População e representatividade.

\begin{tabular}{|c|c|c|c|c|c|c|c|c|}
\hline \multirow{2}{*}{ Elo da cadeia } & \multicolumn{2}{|c|}{ Categoria A } & \multicolumn{2}{|c|}{ Categoria B } & \multicolumn{2}{|c|}{ Categoria C } & \multicolumn{2}{|c|}{ Total } \\
\hline & Qtdade & $\%$ Cntr & Qtdade & $\%$ Cntr & Qtdade & $\%$ Cntr & Qtdade & $\%$ Cntr \\
\hline Clientes de carga & 53 & $59 \%$ & 167 & $26 \%$ & 750 & $15 \%$ & 970 & $100 \%$ \\
\hline Agentes de carga & 36 & $88 \%$ & 43 & $11 \%$ & 61 & $1 \%$ & 140 & $100 \%$ \\
\hline Armadores & 6 & $92 \%$ & 4 & $7 \%$ & 3 & $1 \%$ & 13 & $100 \%$ \\
\hline Operadores logísticos & 17 & $65 \%$ & 84 & $33 \%$ & 217 & $2 \%$ & 318 & $100 \%$ \\
\hline Total & \multicolumn{2}{|l|}{112} & \multicolumn{2}{|l|}{298} & \multicolumn{2}{|l|}{1031} & \multicolumn{2}{|l|}{1441} \\
\hline
\end{tabular}

Fonte: Elaborado pelo autor com dados da área comercial do Tecon RG S.A (2012).

\section{Discussão}

Como a cadeia de suprimentos pode envolver uma grande quantidade de fornecedores, distribuidores, varejistas e outros parceiros, é importante que os gestores tenham formas de determinar onde investir seus esforços. $\mathrm{O}$ método apresentado nesse artigo é uma das formas que as empresas podem utilizar para resolver esse problema. O método apresentado é estruturado de forma a conduzir à uma processo de reflexão dos elementos que influenciam o desempenho da empresa e que estão relacionados com a cadeia de suprimentos. Além disso, é desenvolvido para que seja validado de forma objetiva dentro da organização.

Esta análise permite que os gestores tenham uma visão mais abrangente do seu negócio, facilitando a tomada de decisão. Isto é possível, pois leva-se em consideração elementos internos e externos à organização conforme apontado por diversos autores (LACKMAN, 2000; WEE, 2001; HANNULA; PIRTTIMÄKI, 2003; SABHERWAL; BECERA-FERNANDEZ, 2011; CUSTIS, 2012) como fatores fundamentais para o desenvolvimento da IM e para a construção de patamares coerentes com a estratégia da empresa no balizamento dos seus relacionamentos chave. O conhecimento de seu mercado e a gestão das informações que cerceiam o negócio de cada empresa são essenciais para sua prosperidade e performance positiva.
Ao desenvolver-se este modelo em direção aos terminais portuários especificamente, pretende-se trazer alternativas de governança que vão além dos terminais portuários em si, mas também alcançando a cadeia portuária em que se encontram. Os terminais de container precisam assumir seu novo papel dentro da cadeia portuária, como constata Robinson (2002), deixando de ser apenas um simples provedor de serviço de movimentação e containers para atuar ativamente como elo central de sua cadeia. A gestão de relacionamentos chave a partir de delimitadores balizados pela Inteligência de mercado passa a ser uma ferramenta extra a este segmento, conforme apresentado neste trabalho, mas que também pode ser estendida a outros segmentos.

Além de que, devido a dinâmica do mercado e dos ambientes em que se encontram, esta não é uma padronização estática e válida para o resto da vida da organização. É necessário que de tempos em tempos os delimitadores sejam revisados, conferindo aos dados externos e internos uma nova análise de acordo com a estratégia da organização.

Com base no modelo apresentado, é possível perceber que organizações, mesmo que de outras indústrias que não do setor portuário, adaptem as avaliações conforme o ambiente em que estejam inseridos e assim desenvolvam delimitadores de relacionamentos chave padronizados de acordo com a necessidade da organização. 


\section{Considerações finais}

Em função da relevância do assunto em torno da gestão aprofundada das informações do ambiente em que as organizações se encontram, percebe-se o crescimento de estudos, conceitos e técnicas voltadas para o tema de Inteligência de mercado. $\mathrm{O}$ presente trabalho contribui para essa literatura ao propor um método de direcionamento dos relacionamentos chave ao longo da cadeia de suprimento, testando esse método, e seus resultados, em um terminal de container e toda sua cadeia de suprimentos.

Outra contribuição do trabalho é apresentar uma visão completa da cadeia de suprimentos. Muitos estudos que tratam da cadeia de suprimentos acabam oferecendo uma visão parcial de um ou outro elemento da cadeia ou, em alguns casos, de díades e até tríades. O estudo apresentado nesse artigo proporcionar uma visão completa da cadeia de suprimentos, mostrando como a mesma pode ser complexa. Porém, apresenta-se também um método para auxiliar na gestão dessa complexidade, conforme descrito no parágrafo anterior.

Uma limitação do trabalho é a validação do método proposto somente em uma empresa do setor portuário. Futuros estudos poderão assumir o mesmo método utilizado no presente trabalho para a inclusão de outras indústrias que também necessitam de uma forma estruturada para o desenvolvimento de delimitadores de relacionamentos chave, proporcionando assim novos testes para o método apresentado aqui.

Outra sugestão para estudos futuros é a identificação de ações a serem aplicadas para cada categoria de relacionamento chave. Desta forma, conceitos que apontem como a administração de esforços de acordo com sua relevância pode influenciar no desempenho das organizações e na melhor administração de seus recursos humanos e financeiros.

Estudos longitudinais também poderiam surgir como conseqüência ao trabalho apresentado, pois através do tempo seria possível apresentar a variação de delimitadores estratégicos a partir de IM. Estas variações poderiam ser justificadas conforme os diferentes cenários em que a organização estiver imbuía no decorrer das aplicações do modelo.

\section{Referências}

CAO, M.; ZHANG, Q. Supply Chain Collaboration: Impact On Collaborative Advantage And Firm Performance. Journal of Operations Management, v. 29, p. 163-180, 2011.
CARBONE, V.; DE MARTINO, M. The Changing Role of Ports in Supply Chain Management: An Empirical Analysis, Maritime Policy Management, v. 30, n. 4, p. 305-320, 2003.

CORSTEN, D.; GRUEN, T.; PEYINGAUS, M. The effects of supplier-to-buyer identification on operational performance-An empirical investigation of inter-organizational identification in automotive relationships. Journal of Operations Management, v. 29, p. 549-560, 2011.

CUSTIS, C. The role of business intelligence within the hospitality industry's information systems strategy: Historical concepts and future trends. Journal of Management Policy and Practice, v. 13, n. 3, p. 82-94, 2012.

DE MARTINO, M.; MORVILLO, A. Activities, resources and inter-organizational relationships: key factors in port competitiveness. Maritime Policy \& Management, v. 35, n. 6, p. 571-589, 2008.

DEPARTAMENTOCOMERCIAL TECON RIO GRANDE. Escritório Comercial de Porto Alegre. Último acesso ao banco de dados em fevereiro de 2012.

DISHMAN, P. L; CALOF, J.L. Competitive intelligence: a multiphasic precedent to marketing strategy. European Journal of Marketing, v. 42, n. 7/8, p. 766-785, 2008.

DUBOIS, A.; HULTHÉN, K.; PEDERSEN, A. Interdependence Within and Among 'Supply Chains'. In: 12nd International IPSERA Conference, Budapest, 2003.

ESPER, T.L.; DEFEE, C.C.; MENTZER, J.T. A framework of supply chain orientation. The International Journal of Logistics Management, v. 21, issue 2, p. 161179, 2010.

HANNULA, M.; PIRTTIMÄKI, V. Business Intelligence - Empirical Study on the Top 50 Finnish Companies. Journal of American Academy of Business, v. 2, p. 593600, 2003.

HAYES, R.H.; WHEELWRIGHT, S.C. Restoring Our Competitive Edge: Competing Through Manufacturing. New York: Wiley, 1984.

HUETE, L.; ROTH, A. V. The industrialization and span of retailbanks' delivery systems. Internat. J. Oper. Prod. Management, 1988 v. 8, n. 3, p. 46-66, 2011.

HULT, G.; TOMAS M.; KETCHEN, DAVID J., JR. ADAMS, GARRY L. MENA, JEANNETTE A. Supply chain orientation and balanced scorecard performance. Journal of Managerial Issues, v. 20, n. 4, 2008. 
IVENS, B.S.; PARDO, C.; SALLE, R; COVA, B. Relationship keyness: The underlying concept for different forms of key relationship management. Industrial Marketing Management, v. 38, p. 513-519, 2009.

KOHLIL, A.K.; JAWORSKI, B.J. Market orientation: The construct, research propositions, and managerial implications. Journal of Marketing, v. 54, p. 1-18, 1990.

LACKMAN, C.; SABAN, K.; LANASA, J. The contribution of market intelligence to tactical and strategic business decision. Marketing intelligence Planning, v. 18, n. 1, p. 6-8, 2000.

LAMBERT, D. M.; COOPER, M.C.; PAGH, D.J.; Supply Chain Management: Implementation issues and research opportunities. The Ohio State University, v. 9, n. 2, 1998.

LIN, Y.; WANG, Y.; YU, C. Investigating the drivers of the innovation in channel integration and supply chain performance: A strategy orientated perspective. Int. J. Production Economics, v. 127, p. 320-332, 2010.

MALONI, M.; BENTON; W.C. Power Influences in the Supply Chain. Journal of Business Logistics, v. 21, n. 1, p. 49-74, 1997.

MENTZER, J. T.; KEEBLER, J. S.; NIX, N. W.; SMITH, C. D.; ZACHARIA, Z. G. Defining supply chain management. Journal of Business Logistics, v. 22, n. 2, p. 1-25, 2001.

MIN, S.; MENTZER, J.T.; LADD, R. A market orientation on supply chain management. Journal of the Academic Marketing Science, v. 35, p. 507-522, 2007.

MIOCEVIC, D.; CRNJAK-KARANOVIC, B. The mediating role of key supplier relationship management practices on supply chain orientation-The organizational buying effectiveness link. Industrial Marketing Management, v. 41, p. 115-124, 2012.

OMAR, A.; DAVIS-SRAMEK, B.; FUGATE, B.S.; MENTZER, J.T. Exploring The Complex Social Processes Of Organizational Change:Supply Chain Orientation From A Manager's Perspective. Journal of Business Logistics, v. 33, n. 1, p. 4-19, 2012.
PARDO, C. Key account management in the industrial field. The account team for na efficient reconfiguration of the supplier-customer relationship. In: 17th Annual IMP Conference Olo, Denmark, 2001.

PORTER, M. E. Competitive advantage - Creating and sustaining superior performance, New York: The Free Press, p. 36, 1984.

ROBINSON, R. Port as elements in value-driven chain systems: the new paradigm. Maritime Policy and Management, v. 25, n. 1, p. 21-40, 2002.

SABHERWAL, R., BECERA-FERNANDEZ, I. Business Intelligence: Practices, Technologies and Management, John Williey \& Sons, 2011.

SKINNER, W. Manufacturing - Missing Link in Corporate Strategy. Harvard Business Review, v. 47, n. 3, 1969.

STANK, T. P.; DAVIS, B.R.; FUGATE, B.S. A Strategic Framework For Supply Chain Oriented Logistics. Journal of Business Logistics, v. 2, n. 26, p. 2158-1592, 2011.

STOCK, G. N., \& TATIKONDA, M. V. The joint influence of technology uncertainty and interorganizational interaction on external technology integration success. Journal of Operations Management, v. 26, n. 1, p. 65-80, 2008.

TEIXEIRA, R.; LACERDA, D. P. Gestão da cadeia de suprimentos: análise dos artigos publicados em alguns periódicos acadêmicos entre os anos de 2004 e 2006. Gestão da Produção, v. 17, n. 1, p. 207-227, 2010.

TONGZON, J.; CHANG, Y.; LEE, S. How supply chain oriented is the port sector? International Journal of Production Economics, v. 122, n. 1, p. 21-34, 2009.

WEE, T.T.T. The use of marketing research and intelligence in strategic planning: key issues and future trends. Marketing intelligence Planning, v. 19, n. 4, p. 245-253, 2001.

Recebido em 12/09/2013. Aceito em 28/07/2014. 